\title{
PROOF OF THE HAMILTONICITY-TRACE CONJECTURE FOR SINGULARLY PERTURBED MARKOV CHAINS
}

\author{
VLADIMIR EJOV, ${ }^{*}$ University of South Australia \\ NELLY LITVAK, ${ }^{* *}$ University of Twente \\ GIANG T. NGUYEN, ${ }^{* * *}$ University of South Australia \\ PETER G. TAYLOR, ${ }^{* * * *}$ University of Melbourne
}

\begin{abstract}
We prove the conjecture formulated in Litvak and Ejov (2009), namely, that the trace of the fundamental matrix of a singularly perturbed Markov chain that corresponds to a stochastic policy feasible for a given graph is minimised at policies corresponding to Hamiltonian cycles.
\end{abstract}

Keywords: Stochastic matrix; Hamiltonian cycle; perturbed Markov chain

2010 Mathematics Subject Classification: Primary 60J10

Secondary $05 \mathrm{C} 45 ; 11 \mathrm{C} 20$

\section{Introduction}

Over the last two centuries, the Hamiltonian cycle problem (HCP) has attracted a lot of attention from mathematicians and computer scientists. Given a graph, the objective of the HCP is to determine whether there exists a cycle that goes through every vertex in the graph exactly once. The traditional approaches to this NP-complete problem have mainly involved tools from discrete mathematics, more specifically, graph theory and combinatorics. In 1994, Filar and Krass [11] employed a new technique, in which they embedded the HCP in a Markov decision process, consequently converting the discrete graph theory problem into a continuous optimisation problem, of which the optimal value helps determine whether the graph in consideration possesses a Hamiltonian cycle or not. This led to various new optimisation models involving, for example, the top-left elements of the fundamental matrices of Markov chains associated with a given graph [2], [3], [7], and, more recently, the determinants of the inverse of the fundamental matrices [5], [9]. These novel reformulations of the Hamiltonian cycle problem led to new and promising algorithms, detailed in [1], [6], [8], [12], and, most recently, [14].

The new approach of embedding the HCP in a Markov decision process led to the optimisation model described in [16]. In [16] the authors showed that instead of minimising

Received 2 October 2008; revision received 8 July 2011.

* Postal address: School of Mathematics and Statistics, University of South Australia, Mawson Lakes campus, Mawson Lakes SA 5095, Australia. Email address: ejovvl@gmail.com

** Postal address: Faculty of Electrical Engineering, Mathematics and Computer Science, Department of Applied Mathematics, University of Twente, PO Box 217, 7500 AE Enschede, The Netherlands.

Email address: n.litvak@ewi.utwente.nl

*** Current address: Département d'informatique, Université Libre de Bruxelles, CP 212, Boulevard du Triomphe, 2 , B-1050 Bruxelles, Belgium. Email address: giang.nguyen@ulb.ac.be

**** Postal address: Department of Mathematics and Statistics, University of Melbourne, Parkville VIC 3010, Australia. Email address: p.taylor@ms.unimelb.edu.au 
top-left elements of the fundamental matrices, one can minimise the traces of the fundamental matrices to determine the Hamiltonicity of a given graph. While it is unclear whether the trace function offers an algorithmic advantage over the top-left element function, the former operator is no doubt more well studied and frequently employed. Moreover, this alternative objective function provides new probabilistic interpretations about the link between the Hamiltonian cycle problem and Markov chains. Apart from the choice of objective functions, there are two other major differences between the results for the top-left element optimisation model and those for the trace optimisation model. First, for the top-left element optimisation model, Hamiltonian cycles are shown to be optimisers over the set of doubly stochastic Markov chains, whereas for the trace optimisation model, this result is extended to the larger set of Markov chains. Second, for the optimality results to hold in the model where the objective function is the top-left element, we need a particular singular perturbation to disguise a potentially multiergodic structure in Markov chains. This is not the case when the trace is optimised: in [16] the authors showed that Hamiltonian cycles, if they exist, optimise the trace function without the necessary employment of a perturbation. They conjectured that the optimality of Hamiltonian cycles for the trace function still holds over the set of Markov chains when we apply a singular perturbation to the Markov chains permissible on a given graph. In this paper we prove this conjecture, consequently demonstrating the robustness of this objective function and increasing the flexibility for future algorithms implementing this optimisation model.

We would like to remark that matrices with linear perturbations and their stationary distributions have attracted a lot of attention in the recent literature, triggered by the problem of node ranking in complex stochastic networks. Specifically, such matrices are used in the Google PageRank algorithm that determines the popularity of Web pages. PageRank is defined as the stationary distribution of a Markov chain on the set of Web pages. At each step, with probability $1-\varepsilon$, a surfer follows a randomly chosen out-going hyperlink of a current page, and, with probability $\varepsilon$, the surfer is bored and picks a new page on the Web at random. After the introduction of PageRank by Brin and Page [4], a great deal of work was carried out on PageRank computation and analysis; see, for example, [15], [19], and the references therein.

The structure of the paper is as follows. In Section 2 we introduce the notation, recall relevant theorems, and present our main result that Hamiltonian cycles are optimisers for the trace function of fundamental matrices of singularly perturbed Markov chains. In Section 3 we provide a proof of this main result that requires several lemmas. We employ an analytic approach that involves eigenvalues and eigenvectors to establish important optimality properties of the trace. In order to obtain the exact expressions for the trace, we apply probabilistic arguments that relate the value of the trace to the occupancy measures of Markov processes with geometric stopping times.

\section{Optimality of Hamiltonian cycles}

Consider a given connected graph $\Gamma$ of $N$ vertices. Let $\boldsymbol{P}$ be an $N \times N$ transition probability matrix corresponding to a feasible policy on $\Gamma$, that is, $p_{i j}=0$ whenever $(i, j)$ is not an edge on $\Gamma$. Furthermore, let $\boldsymbol{J}$ be an $N \times N$ matrix, every element of which is unity. As every row of $\boldsymbol{P}$ sums to $1, \boldsymbol{P}$ is said to be stochastic. If every column of $\boldsymbol{P}$ also sums up to $1, \boldsymbol{P}$ is said to be doubly stochastic. A policy is considered deterministic if $\boldsymbol{P}$ contains only $1 \mathrm{~s}$ and $0 \mathrm{~s}$, and randomised otherwise. We denote the sets of stochastic policies and doubly stochastic policies on $\Gamma$ respectively by $\&(\Gamma)$ and $\mathscr{D} \&(\Gamma)$, and a policy corresponding to a Hamiltonian cycle on 
the graph as $\boldsymbol{P}_{\mathrm{HC}}$. Define $\boldsymbol{P}(\varepsilon):=\boldsymbol{P}^{\varepsilon}$ to be the following linear symmetric perturbation of $\boldsymbol{P}$ :

$$
\boldsymbol{P}^{\varepsilon}:=(1-\varepsilon) \boldsymbol{P}+\frac{\varepsilon}{N} \boldsymbol{J} .
$$

Let $\Pi^{*}(\boldsymbol{P}, \varepsilon)$ be the stationary distribution matrix of the Markov chain specified by $\boldsymbol{P}^{\varepsilon}$, i.e.

$$
\boldsymbol{\Pi}^{*}(\boldsymbol{P}, \varepsilon):=\lim _{t \rightarrow \infty}\left(\boldsymbol{P}^{\varepsilon}\right)^{t},
$$

and let $\boldsymbol{G}(\boldsymbol{P}, \varepsilon)$ to be the corresponding fundamental matrix:

$$
\boldsymbol{G}(\boldsymbol{P}, \varepsilon):=\left(\boldsymbol{I}-\boldsymbol{P}^{\varepsilon}+\boldsymbol{\Pi}^{*}(\boldsymbol{P}, \varepsilon)\right)^{-1} .
$$

For notational convenience, we write

$$
\boldsymbol{A}_{*}(\boldsymbol{P}, \varepsilon):=\boldsymbol{I}-\boldsymbol{P}^{\varepsilon}+\boldsymbol{\Pi}^{*}(\boldsymbol{P}, \varepsilon)
$$

and

$$
\boldsymbol{A}(\boldsymbol{P}, \varepsilon):=\boldsymbol{I}-\boldsymbol{P}^{\varepsilon}+\frac{1}{N} \boldsymbol{J}
$$

The notation $\operatorname{tr}[\boldsymbol{A}]$ and $\operatorname{det} \boldsymbol{A}$ denote the trace and the determinant of a matrix $\boldsymbol{A}$, respectively. We recall here some relevant results connecting the fundamental matrix $\boldsymbol{G}(\boldsymbol{P}, \varepsilon)$ and the matrix $\boldsymbol{A}(\boldsymbol{P}, \varepsilon)$ to the Hamiltonicity of a given graph.

Theorem 1. ([5], [9].) For a Hamiltonian graph $\Gamma$ and any $\varepsilon \in[0,1)$,

$$
\max _{\boldsymbol{P} \in S(\Gamma)} \operatorname{det} \boldsymbol{A}(\boldsymbol{P}, \varepsilon)=\operatorname{det} \boldsymbol{A}\left(\boldsymbol{P}_{\mathrm{HC}}, \varepsilon\right) .
$$

In particular, for any Hamiltonian policy $\boldsymbol{P}_{\mathrm{HC}}$ permissible on the graph,

$$
\operatorname{det} \boldsymbol{A}\left(\boldsymbol{P}_{\mathrm{HC}}, \varepsilon\right)= \begin{cases}N & \text { if } \varepsilon=0, \\ \frac{1-(1-\varepsilon)^{N}}{\varepsilon} & \text { if } \varepsilon \in(0,1) .\end{cases}
$$

In [16] the authors proved that, for $\varepsilon \in[0,1)$, the minimisers of $\operatorname{tr}[\boldsymbol{G}(\boldsymbol{P}, \varepsilon)]$ over the set of doubly stochastic policies $\mathscr{D} \&(\Gamma)$ correspond to Hamiltonian cycles. They also showed that this result holds over the set of stochastic policies for $\varepsilon=0$, that is, without any perturbation.

Theorem 2. ([16].) For a given Hamiltonian graph $\Gamma$,

$$
\min _{\boldsymbol{P} \in \delta(\Gamma)} \operatorname{tr}[\boldsymbol{G}(\boldsymbol{P}, 0)]=\operatorname{tr}\left[\boldsymbol{G}\left(\boldsymbol{P}_{\mathrm{HC}}, 0\right)\right]
$$

for any $\boldsymbol{P}_{\mathrm{HC}}$ corresponding to a Hamiltonian cycle on the graph.

In [16] the authors also included a conjecture extending this result to $\varepsilon \in[0,1)$.

Conjecture 1. (Hamiltonicity-trace conjecture [16].) For a given Hamiltonian graph $\Gamma$ and any $\varepsilon \in[0,1)$,

$$
\min _{\boldsymbol{P} \in \delta(\Gamma)} \operatorname{tr}[\boldsymbol{G}(\boldsymbol{P}, \varepsilon)]=\operatorname{tr}\left[\boldsymbol{G}\left(\boldsymbol{P}_{\mathrm{HC}}, \varepsilon\right)\right]
$$

for any $\boldsymbol{P}_{\mathrm{HC}}$ corresponding to a Hamiltonian cycle. 
The main result of this paper is as follows.

Theorem 3. For a given Hamiltonian graph $\Gamma$ and any $\varepsilon \in(0,1)$,

$$
\min _{\boldsymbol{P} \in \mathcal{S}(\Gamma)} \operatorname{tr}[\boldsymbol{G}(\boldsymbol{P}, \varepsilon)]=\operatorname{tr}\left[\boldsymbol{G}\left(\boldsymbol{P}_{\mathrm{HC}}, \varepsilon\right)\right]=1+\frac{\varepsilon N-\left(1-(1-\varepsilon)^{N}\right)}{\varepsilon\left(1-(1-\varepsilon)^{N}\right)}
$$

for any $\boldsymbol{P}_{\mathrm{HC}}$ corresponding to a Hamiltonian cycle.

The proof of Theorem 3 is presented in the next section. It has the following structure. First, in Lemma 1 below, we establish the relationships between eigenvalues and eigenvectors of the matrix $\boldsymbol{P}$ and a particular matrix function of $\boldsymbol{P}$. Unless stated otherwise, the terms eigenvectors refer to right eigenvectors. The result of Lemma 1 gives rise to alternative formulae for the trace function, which we enunciate in Corollary 1 below. Furthermore, in Lemma 2 below, we prove that the trace of the fundamental matrix $\boldsymbol{G}(\boldsymbol{P}, \varepsilon)$ when $\varepsilon \in(0,1)$ for any randomised policy is bounded above by that of some deterministic policy, and bounded below by that of some other deterministic policy. This enables us to reduce our proof from the set of all stochastic policies to the set of all deterministic policies only. We derive the exact formulae for four exhaustive, mutually exclusive types of deterministic policy in Lemmas 3-6 below. Finally, we show that among these, Hamiltonian cycles are minimisers for the objective function.

\section{Proof of the main result}

Let $\boldsymbol{Q}$ and $\boldsymbol{W}$ be arbitrary but fixed $N \times N$ rank-one stochastic matrices, that is, $\boldsymbol{Q}:=\boldsymbol{e q}$ and $\boldsymbol{W}:=\boldsymbol{e} \boldsymbol{w}$ for some vectors $\boldsymbol{q}$ and $\boldsymbol{w}$ such that $\boldsymbol{q}, \boldsymbol{w} \geq \mathbf{0}, \boldsymbol{q} \boldsymbol{e}=\mathbf{1}$, and $\boldsymbol{w} \boldsymbol{e}=\mathbf{1}$, where $\boldsymbol{e}=$ $(1, \ldots, 1)^{\top}$. Define two matrices $\boldsymbol{P}_{\boldsymbol{Q}}(\varepsilon):=(1-\varepsilon) \boldsymbol{P}+\varepsilon \boldsymbol{Q}$ and $\boldsymbol{A}_{\boldsymbol{W}}\left(\boldsymbol{P}_{\boldsymbol{Q}}(\varepsilon)\right):=\boldsymbol{I}-\boldsymbol{P}_{\boldsymbol{Q}}(\varepsilon)+\boldsymbol{W}$. For $i=1, \ldots, N$, we denote the eigenvalues of $\boldsymbol{P}$ by $\lambda_{i}$, the eigenvalues of $\boldsymbol{P}_{\boldsymbol{Q}}(\varepsilon)$ by $\eta_{i}$, and the stationary vector of $\boldsymbol{A}_{W}\left(\boldsymbol{P}_{\boldsymbol{Q}}(\varepsilon)\right)$ by $\boldsymbol{s}$, that is, $\boldsymbol{s} \boldsymbol{A}_{\boldsymbol{W}}\left(\boldsymbol{P}_{\boldsymbol{Q}}(\varepsilon)\right)=\boldsymbol{s}$ and $\boldsymbol{s} \boldsymbol{e}=1$. Thus, $\boldsymbol{s} \boldsymbol{P}=(1-\varepsilon)^{-1}(\boldsymbol{w}-\varepsilon \boldsymbol{q})$.

The relationship between the $\lambda_{i}$ and the $\eta_{i}$ was given in Theorem 2.3 of [18]. Specifically,

$$
\eta_{i}= \begin{cases}(1-\varepsilon) \lambda_{i} & \text { for } i=1, \ldots, N-1, \\ \lambda_{N}=1 & \text { for } i=N .\end{cases}
$$

The following lemma gives the relationship between the eigenvalues and eigenvectors of $\boldsymbol{P}_{\boldsymbol{Q}}(\varepsilon)$ and the eigenvalues and eigenvectors of $\boldsymbol{A}_{W}\left(\boldsymbol{P}_{\boldsymbol{Q}}(\varepsilon)\right)$.

Lemma 1. For $\varepsilon \in(0,1)$ and any stochastic matrix $\boldsymbol{P}$, every eigenvector $\boldsymbol{v}_{i}$ of $\boldsymbol{P}_{\boldsymbol{Q}}(\varepsilon)$ corresponding to the eigenvalue $\eta_{i}<1$ gives an eigenvector $(\boldsymbol{I}-\boldsymbol{e s}) \boldsymbol{v}_{i}$ of $\boldsymbol{A}_{\boldsymbol{W}}\left(\boldsymbol{P}_{\boldsymbol{Q}}(\varepsilon)\right)$ corresponding to the eigenvalue $1-\eta_{i}$. The eigenvector $\boldsymbol{e}$ of $\boldsymbol{P}_{\boldsymbol{Q}}(\varepsilon)$ corresponding to the eigenvalue 1 is an eigenvector of $\boldsymbol{A}_{W}\left(\boldsymbol{P}_{\boldsymbol{Q}}(\varepsilon)\right)$ corresponding to the eigenvalue 1.

Proof. Because $\boldsymbol{P}_{\boldsymbol{Q}}(\varepsilon)$ is irreducible, the Perron-Frobenius theorem (see, for example, [17]) guarantees that its maximal eigenvalue 1 is simple and we can write its Jordan canonical form $\boldsymbol{L}$ as

$$
\boldsymbol{L}=\left[\begin{array}{l}
\boldsymbol{U} \\
\boldsymbol{\pi}
\end{array}\right] \boldsymbol{P}_{\boldsymbol{Q}}(\varepsilon)[\boldsymbol{V}, \boldsymbol{e}],
$$

where $\boldsymbol{U} \boldsymbol{e}=\mathbf{0}, \boldsymbol{\pi} \boldsymbol{P}_{\boldsymbol{Q}}(\varepsilon)=\boldsymbol{\pi}, \boldsymbol{\pi} \boldsymbol{e}=1, \boldsymbol{\pi} \boldsymbol{V}=\mathbf{0}, \boldsymbol{U} \boldsymbol{V}=\boldsymbol{I}_{N-1}$, and $\boldsymbol{V} \boldsymbol{U}=\boldsymbol{I}_{N}-\boldsymbol{e} \boldsymbol{\pi}$. Then, it follows in a straightforward way that

$$
\boldsymbol{L}=\left[\begin{array}{cc}
\boldsymbol{U} \boldsymbol{P}_{\boldsymbol{Q}^{(\varepsilon)}} \boldsymbol{V} & \mathbf{0} \\
\mathbf{0} & 1
\end{array}\right]
$$


Let $\boldsymbol{C}$ be a Jordan canonical block with the same structure as that of $\boldsymbol{L}$, with eigenvalues $1-\eta_{i}$ for $i=1, \ldots, N-1$ and the last eigenvalue being 1 . We now show that $\boldsymbol{C}$ is the Jordan canonical form of $\boldsymbol{A}_{\boldsymbol{W}}\left(\boldsymbol{P}_{\boldsymbol{Q}}(\varepsilon)\right)$, that is,

$$
\boldsymbol{C}=\left[\begin{array}{l}
\boldsymbol{U} \\
\boldsymbol{s}
\end{array}\right] \boldsymbol{A}_{\boldsymbol{W}}\left(\boldsymbol{P}_{\boldsymbol{Q}}(\varepsilon)\right)[\boldsymbol{V}-\boldsymbol{e s} \boldsymbol{V}, \boldsymbol{e}] .
$$

Expanding the right-hand side of (3.2) gives

$$
\begin{aligned}
{\left[\begin{array}{c}
\boldsymbol{U} \\
\boldsymbol{s}
\end{array}\right] } & \left(\boldsymbol{I}-\boldsymbol{P}_{\boldsymbol{Q}}(\varepsilon)+\boldsymbol{W}\right)[\boldsymbol{V}-\boldsymbol{e s} \boldsymbol{V}, \boldsymbol{e}] \\
= & {\left[\begin{array}{cr}
\boldsymbol{U}\left(\boldsymbol{I}-\boldsymbol{P}_{\boldsymbol{Q}}(\varepsilon)+\boldsymbol{W}\right) \boldsymbol{V} & \mathbf{0} \\
\boldsymbol{s}(\boldsymbol{V}-\boldsymbol{e s} \boldsymbol{V}) & 1
\end{array}\right] } \\
= & {\left[\begin{array}{cc}
\boldsymbol{I}-\boldsymbol{U} \boldsymbol{P}_{\boldsymbol{Q}}(\varepsilon) \boldsymbol{V} & \mathbf{0} \\
\boldsymbol{s}\left(\boldsymbol{I}-\boldsymbol{P}_{\boldsymbol{Q}}(\varepsilon)+\boldsymbol{W}\right)(\boldsymbol{V}-\boldsymbol{e s} \boldsymbol{V}) & 1
\end{array}\right] . }
\end{aligned}
$$

Focusing on the bottom-left entry of the above right-hand side, we have

$$
\begin{aligned}
\boldsymbol{s}\left(\boldsymbol{I}-\boldsymbol{P}_{\boldsymbol{Q}}(\varepsilon)+\boldsymbol{W}\right)(\boldsymbol{V}-\boldsymbol{e s} \boldsymbol{V}) & =\boldsymbol{s}(\boldsymbol{V}-(1-\varepsilon) \boldsymbol{P} \boldsymbol{V}-\varepsilon \boldsymbol{e q} \boldsymbol{V}+\boldsymbol{e w} \boldsymbol{V}-\boldsymbol{e s} \boldsymbol{V}) \\
& =\boldsymbol{s} \boldsymbol{V}-(\boldsymbol{w}-\varepsilon \boldsymbol{q}) \boldsymbol{V}-\varepsilon \boldsymbol{q} \boldsymbol{V}+\boldsymbol{w} \boldsymbol{V}-\boldsymbol{s} \boldsymbol{V} \\
& =0,
\end{aligned}
$$

which proves the lemma.

Let $\boldsymbol{t}$ and $\boldsymbol{r}$ be the respective stationary vectors of $\boldsymbol{A}(\boldsymbol{P}, \varepsilon)$ and $\boldsymbol{A}_{*}(\boldsymbol{P}, \varepsilon)$, that is, $\boldsymbol{t} \boldsymbol{A}(\boldsymbol{P}, \varepsilon)=$ $\boldsymbol{t}, \boldsymbol{r} \boldsymbol{A}_{*}(\boldsymbol{P}, \varepsilon)=\boldsymbol{r}$, and $\boldsymbol{t} \boldsymbol{e}=\boldsymbol{r} \boldsymbol{e}=1$. We now easily obtain the eigenvalues and eigenvectors of the matrices $\boldsymbol{A}(\boldsymbol{P}, \varepsilon), \boldsymbol{A}_{*}(\boldsymbol{P}, \varepsilon)$, and $\boldsymbol{G}(\boldsymbol{P}, \varepsilon)$.

Corollary 1. For $\varepsilon \in(0,1)$ and any stochastic matrix $\boldsymbol{P}$, the following statements hold.

(i) For $i=1, \ldots, N-1$, every eigenvector $\boldsymbol{v}_{i}$ of $\boldsymbol{P}^{\varepsilon}$ corresponding to the eigenvalue $\eta_{i}<1$ gives an eigenvector $(\boldsymbol{I}-\boldsymbol{e t}) \boldsymbol{v}_{i}$ or $(\boldsymbol{I}-\boldsymbol{e r}) \boldsymbol{v}_{i}$ of $\boldsymbol{A}(\boldsymbol{P}, \varepsilon)$ or $\boldsymbol{A}_{*}(\boldsymbol{P}, \varepsilon)$, respectively, corresponding to the eigenvalue $1-\eta_{i}$. The eigenvector $\boldsymbol{e}:=(1, \ldots, 1)^{\top}$ of $\boldsymbol{P}$ corresponding to the unique eigenvalue 1 is an eigenvector of $\boldsymbol{A}(\boldsymbol{P}, \varepsilon)$ or $\boldsymbol{A}_{*}(\boldsymbol{P}, \varepsilon)$ corresponding to the eigenvalue 1.

(ii) For $i=1, \ldots, N-1$, the eigenvalues of $\boldsymbol{G}(\boldsymbol{P}, \varepsilon)$ are 1 and $1 /\left(1-(1-\varepsilon) \lambda_{i}\right)$.

(iii) $\operatorname{tr}[\boldsymbol{G}(\boldsymbol{P}, \varepsilon)]=1+\sum_{i=1}^{N-1} 1 /\left(1-(1-\varepsilon) \lambda_{i}\right)=\operatorname{tr}\left[(\boldsymbol{I}-(1-\varepsilon) \boldsymbol{P})^{-1}\right]-(1-\varepsilon) / \varepsilon$.

Proof. Part (i) follows from Lemma 1 by setting $\boldsymbol{Q}=1 / N \boldsymbol{J}$ and $\boldsymbol{W}=1 / N \boldsymbol{J}$ or, respectively, $\boldsymbol{W}=\Pi^{*}(\boldsymbol{P}, \varepsilon)$. We obtain (ii) and (iii) by (3.1), and the well-known properties that the eigenvalues of an invertible matrix are the reciprocals of the eigenvalues of its inverse and that the trace of a matrix is the sum of its eigenvalues. In the last equality of (iii), we note that the eigenvalues of $(\boldsymbol{I}-(1-\varepsilon) \boldsymbol{P})$ are $\varepsilon$ and, for $i=1, \ldots, N-1,1-(1-\varepsilon) \lambda_{i}$.

Remark 1. It is easy to check that Corollary 1 holds for general rank-one stochastic matrices $\boldsymbol{Q}$ and $\boldsymbol{W}$. For the proof of a generalised version of Corollary 1(iii), see the preprint version of this paper [10].

Lemma 1 implies that the eigenvalues of $\boldsymbol{A}_{\boldsymbol{W}}\left(\boldsymbol{P}_{\boldsymbol{Q}}(\varepsilon)\right)$ do not depend on $\boldsymbol{W}$ and $\boldsymbol{Q}$. For this fact, the preprint version of this paper [10] offers a simple probabilistic interpretation, 
which is related to the properties of the fundamental matrix of a positive recurrent Markov chain [13, p. 459]. This observation is crucial for our further discussion because instead of $\operatorname{tr}[\boldsymbol{G}(\boldsymbol{P}, \varepsilon)]=\operatorname{tr}\left[\boldsymbol{A}_{*}^{-1}(\boldsymbol{P}, \varepsilon)\right]$ we may consider $\operatorname{tr}\left[\boldsymbol{A}^{-1}(\boldsymbol{P}, \varepsilon)\right]$, which is beneficial because the elements of the matrix $\boldsymbol{A}(\boldsymbol{P}, \varepsilon)$ depend linearly on the elements of $\boldsymbol{P}$. This allows us to take the next step in the proof of Theorem 3, by stating that the trace of the fundamental matrix can be maximised or minimised only at Markov chains associated with deterministic policies. This is formalised in the following lemma.

Lemma 2. For any $\varepsilon \in(0,1)$ and every randomised policy $\boldsymbol{P}$, there exist some deterministic policies $\boldsymbol{D}_{1}$ and $\boldsymbol{D}_{2}$ such that

$$
\operatorname{tr}\left[\boldsymbol{G}\left(\boldsymbol{D}_{1}, \varepsilon\right)\right] \leq \operatorname{tr}[\boldsymbol{G}(\boldsymbol{P}, \varepsilon)] \leq \operatorname{tr}\left[\boldsymbol{G}\left(\boldsymbol{D}_{2}, \varepsilon\right)\right] .
$$

Proof. Let $\boldsymbol{P}$ be a randomised policy, and consider the randomisation at each row $i$ of $\boldsymbol{P}$ separately. Suppose that a particular row $i$ has the structure

$$
\left[\begin{array}{lllllll}
\cdots & a & \cdots & b & \cdots & c & \cdots
\end{array}\right]
$$

where $a, b \in(0,1)$ and $a+b+c=1$. Note that the subsequent arguments are easily generalizable to the case with more than three nonzero entries of the row in consideration. Consider a policy $\boldsymbol{P}_{\gamma}$ that coincides with $\boldsymbol{P}$ in all rows except row $i$, where it is replaced by

$$
\left[\begin{array}{lllllll}
\cdots & \gamma & \cdots & \frac{(1-\gamma)}{1-a} b & \cdots & \frac{(1-\gamma)}{1-a} c & \cdots
\end{array}\right], \quad \gamma \in[0,1] .
$$

Note that, for $\gamma=a$, the matrix $\boldsymbol{P}_{\gamma}$ reduces to $\boldsymbol{P}$. By Corollary 1 and writing the inverse in terms of the adjoint,

$$
\operatorname{tr}\left[\boldsymbol{G}\left(\boldsymbol{P}_{\gamma}, \varepsilon\right)\right]=\operatorname{tr}\left[\boldsymbol{A}^{-1}\left(\boldsymbol{P}_{\gamma}, \varepsilon\right)\right]=\sum_{i=1}^{N} \frac{\operatorname{det} \boldsymbol{A}_{i i}\left(\boldsymbol{P}_{\gamma}, \varepsilon\right)}{\operatorname{det} \boldsymbol{A}\left(\boldsymbol{P}_{\gamma}, \varepsilon\right)},
$$

where $\boldsymbol{A}\left(\boldsymbol{P}_{\gamma}, \varepsilon\right)=\boldsymbol{I}-\boldsymbol{P}_{\gamma}^{\varepsilon}+1 / N \boldsymbol{J}$ and $\boldsymbol{A}_{i i}\left(\boldsymbol{P}_{\gamma}, \varepsilon\right)$ is the matrix $\boldsymbol{A}\left(\boldsymbol{P}_{\gamma}, \varepsilon\right)$ with its $i$ th row and $i$ th column removed. As both $\operatorname{det} \boldsymbol{A}\left(\boldsymbol{P}_{\gamma}, \varepsilon\right)$ and $\operatorname{det} \boldsymbol{A}_{i i}\left(\boldsymbol{P}_{\gamma}, \varepsilon\right)$ are linear functions of $\gamma$ for all $i=1, \ldots, N$, we have

$$
\operatorname{tr}\left[\boldsymbol{G}\left(\boldsymbol{P}_{\gamma}, \varepsilon\right)\right]=\frac{C_{1}\left(\operatorname{det} \boldsymbol{A}\left(\boldsymbol{P}_{\gamma}, \varepsilon\right)\right)+C_{2}}{\operatorname{det} \boldsymbol{A}\left(\boldsymbol{P}_{\gamma}, \varepsilon\right)}=C_{1}+\frac{C_{2}}{\operatorname{det} \boldsymbol{A}\left(\boldsymbol{P}_{\gamma}, \varepsilon\right)}
$$

for some constants $C_{1}$ and $C_{2}, C_{1} \neq 0$. Differentiating the objective function with respect to $\gamma$ gives

$$
\frac{\partial}{\partial \gamma} \operatorname{tr}\left[\boldsymbol{G}\left(\boldsymbol{P}_{\gamma}, \varepsilon\right)\right]=-\frac{C_{3}}{\left(\operatorname{det} \boldsymbol{A}\left(\boldsymbol{P}_{\gamma}, \varepsilon\right)\right)^{2}},
$$

which is either 0 for all $\gamma \in(0,1)$ if $C_{3}=0$, or never 0 for all $\gamma \in(0,1)$ if $C_{3} \neq 0$. In both cases, this implies that $\operatorname{tr}\left[\boldsymbol{G}\left(\boldsymbol{P}_{\gamma}, \varepsilon\right)\right]$ is a monotone function over $\gamma \in[0,1]$, and is maximised or minimised at either extreme of the interval. As the $i$ th row in $\boldsymbol{P}_{\gamma=0}$ or $\boldsymbol{P}_{\gamma=1}$ has at least one more 0 than the $i$ th row in $\boldsymbol{P}, \boldsymbol{P}_{\gamma=0}$ or $\boldsymbol{P}_{\gamma=1}$ has at least one more 0 than $\boldsymbol{P}$, and either

1. $\operatorname{tr}\left[\boldsymbol{G}\left(\boldsymbol{P}_{\gamma=0}, \varepsilon\right)\right]$ or $\operatorname{tr}\left[\boldsymbol{G}\left(\boldsymbol{P}_{\gamma=1}, \varepsilon\right)\right] \geq \operatorname{tr}[\boldsymbol{G}(\boldsymbol{P}, \varepsilon)]$, or

2. $\operatorname{tr}\left[\boldsymbol{G}\left(\boldsymbol{P}_{\gamma=1}, \varepsilon\right)\right]$ or $\operatorname{tr}\left[\boldsymbol{G}\left(\boldsymbol{P}_{\gamma=0}, \varepsilon\right)\right] \leq \operatorname{tr}[\boldsymbol{G}(\boldsymbol{P}, \varepsilon)]$,

respectively. 
Applying this process of increasing the number of 0 s and consequently reducing the number of randomisations, we can find two deterministic policies $\boldsymbol{D}_{1}$ and $\boldsymbol{D}_{2}$ that satisfy the inequalities in (3.3).

It follows from Lemma 2 that in order to prove Theorem 3, it is sufficient to compare $\operatorname{tr}\left[\boldsymbol{G}\left(\boldsymbol{P}_{\mathrm{HC}}, \varepsilon\right)\right]$ to the values of $\operatorname{tr}[\boldsymbol{G}(\boldsymbol{P}, \varepsilon)]$, where $\boldsymbol{P}$ induces a deterministic policy. To this end, in Lemma 3 below we derive the expression for $\operatorname{tr}\left[\boldsymbol{G}\left(\boldsymbol{P}_{\mathrm{HC}}, \varepsilon\right)\right]$ and then, in Lemmas 4-6 below, we derive the expression for $\operatorname{tr}[\boldsymbol{G}(\boldsymbol{P}, \varepsilon)]$ corresponding to three other exhaustive mutually exclusive classes of deterministic policies.

Lemma 3. For any $\varepsilon \in(0,1)$ and any $\boldsymbol{P}_{\mathrm{HC}}$ that corresponds to a Hamiltonian cycle, that is, a policy with a single ergodic class and no transient states,

$$
\operatorname{tr}\left[\boldsymbol{G}\left(\boldsymbol{P}_{\mathrm{HC}}, \varepsilon\right)\right]=1+\frac{\varepsilon N-\left(1-(1-\varepsilon)^{N}\right)}{\varepsilon\left(1-(1-\varepsilon)^{N}\right)} .
$$

Proof. Consider a random walk that starts at $i$ and is governed by $\boldsymbol{P}$. The random walk governed by the strictly substochastic matrix $(1-\varepsilon) \boldsymbol{P}$ is a 'stopped version' of this random walk in which at each time step there is a probability $\varepsilon$ of termination. The termination time $T(\varepsilon)$ is geometrically distributed with parameter $\varepsilon$. For $i=1, \ldots, N$, the $(i, i)$ th entry of $[\boldsymbol{I}-(1-\varepsilon) \boldsymbol{P}]^{-1}$ is equal to the expected number of visits to $i$ on $[0, T(\varepsilon)]$ given that the random walk starts in state $i$. Now assume that $\boldsymbol{P}=\boldsymbol{P}_{\mathrm{HC}}$. Then the random walk proceeds in cycles of length $N$, and, thus, starting from any state $i$, the probability that it ever returns to $i$ is $(1-\varepsilon)^{N}$, implying that the expected number of returns is $\left(1-(1-\varepsilon)^{N}\right)^{-1}$. Hence, from Corollary 1(iii) we obtain

$$
\operatorname{tr}\left[\boldsymbol{G}\left(\boldsymbol{P}_{\mathrm{HC}}, \varepsilon\right)\right]=\frac{N}{1-(1-\varepsilon)^{N}}-\frac{1-\varepsilon}{\varepsilon}=1+\frac{\varepsilon N-\left(1-(1-\varepsilon)^{N}\right)}{\varepsilon\left(1-(1-\varepsilon)^{N}\right)} .
$$

Lemma 4. For $\varepsilon \in(0,1)$ and any $\boldsymbol{P}$ that corresponds to a deterministic policy with $\ell>1$ ergodic classes and no transient states,

$$
\operatorname{tr}[\boldsymbol{G}(\boldsymbol{P}, \varepsilon)]=1+\frac{\ell-1}{\varepsilon}+\sum_{i=1}^{\ell}\left[\frac{m_{i} \varepsilon-\left(1-(1-\varepsilon)^{m_{i}}\right)}{\varepsilon\left(1-(1-\varepsilon)^{m_{i}}\right)}\right]
$$

where $m_{i}$ is the size of the ith ergodic class in $\boldsymbol{P}$.

Proof. Consider again a diagonal element of $[\boldsymbol{I}-(1-\varepsilon) \boldsymbol{P}]^{-1}$. If there are $\ell$ ergodic classes then the Markov chain given by $\boldsymbol{P}$ splits into separate cycles of lengths $m_{1}, \ldots, m_{\ell}$. For each of the cycles, we can apply the argument from the proof of Lemma 3. Then a diagonal element that corresponds to a state in ergodic class $i$ equals $1 /\left(1-(1-\varepsilon)^{m_{i}}\right)$. Summing over all diagonal elements and using Corollary 1(iii), we derive

$$
\operatorname{tr}\left[\boldsymbol{G}\left(\boldsymbol{P}_{\mathrm{HC}}, \varepsilon\right)\right]=\sum_{i=1}^{\ell} \frac{m_{i}}{1-(1-\varepsilon)^{m_{i}}}-\frac{1-\varepsilon}{\varepsilon}=1+\frac{\ell-1}{\varepsilon}+\sum_{i=1}^{\ell}\left[\frac{m_{i} \varepsilon-\left(1-(1-\varepsilon)^{m_{i}}\right)}{\varepsilon\left(1-(1-\varepsilon)^{m_{i}}\right)}\right] .
$$

To obtain the last equation, it is sufficient to subtract and add $(\ell-1)(1-\varepsilon) / \varepsilon$ in the second expression and then use the result of Lemma 3. 
Lemma 5. For any $\varepsilon \in(0,1)$ and any $\boldsymbol{P}$ that corresponds to a policy with a single ergodic class and one or more transient states,

$$
\operatorname{tr}[\boldsymbol{G}(\boldsymbol{P}, \varepsilon)]=N-m+1+\frac{m \varepsilon-\left(1-(1-\varepsilon)^{m}\right)}{\varepsilon\left(1-(1-\varepsilon)^{m}\right)},
$$

where $m<N$ is the size of the single ergodic class.

Proof. Let $i$ be a transient state of the Markov chain with transition matrix $(1-\varepsilon) \boldsymbol{P}$. Since $\boldsymbol{P}$ is deterministic, a Markov random walk with transition started in $i$ can never return to $i$. Recalling that the $(i, i)$ th element of $[\boldsymbol{I}-(1-\varepsilon) \boldsymbol{P}]^{-1}$ is the average number of visits to $i$ on $[0, T(\varepsilon)]$ starting from $i$, we conclude that each transient state contributes precisely one to $\operatorname{tr}\left[[\boldsymbol{I}-(1-\varepsilon) \boldsymbol{P}]^{-1}\right]$. On the other hand, ergodic states form a cycle of length $m$, and we can compute the contribution of these states by applying the argument as in the proof of Lemma 3 with $N=m$. Summing the contributions of transient and ergodic states and applying Corollary 1(iii) we obtain the result of the lemma.

Lemma 6. For any $\varepsilon \in(0,1)$ and any $\boldsymbol{P}$ corresponding to a policy with multiple ergodic classes and one or more transient states,

$$
\operatorname{tr}[\boldsymbol{G}(\boldsymbol{P}, \varepsilon)]=N-\sum_{i=1}^{\ell} m_{i}+1+\frac{\ell-1}{\varepsilon}+\sum_{i=1}^{\ell}\left[\frac{m_{i} \varepsilon-\left(1-(1-\varepsilon)^{m_{i}}\right)}{\varepsilon\left(1-(1-\varepsilon)^{m_{i}}\right)}\right],
$$

where $m_{i}$ is the size of the ith ergodic class in $\boldsymbol{P}$.

Proof. The result follows by combining the proofs of Lemmas 4 and 5.

Now we are ready to prove Theorem 3.

Proof of Theorem 3. We need to show that, for any $\varepsilon \in(0,1)$ and any stochastic policy $\boldsymbol{P}$ feasible on a given Hamiltonian graph, Hamiltonian cycles are indeed the minimisers.

As the result of Lemma 2 enables us to reduce the proof for the set of stochastic policies to the proof for the set of deterministic policies, by Lemmas 3, 4, 5, and 6, all we need to show now is that, for $\ell>1$ and $m, m_{i}<N$ with $\sum_{i=1}^{\ell} m_{i} \leq N$,

$$
\begin{aligned}
1+\frac{\varepsilon N-\left(1-(1-\varepsilon)^{N}\right)}{\varepsilon\left(1-(1-\varepsilon)^{N}\right)} \leq & 1+\frac{\ell-1}{\varepsilon}+\sum_{i=1}^{\ell}\left[\frac{m_{i} \varepsilon-\left(1-(1-\varepsilon)^{m_{i}}\right)}{\varepsilon\left(1-(1-\varepsilon)^{m_{i}}\right)}\right] \\
1+\frac{\varepsilon N-\left(1-(1-\varepsilon)^{N}\right)}{\varepsilon\left(1-(1-\varepsilon)^{N}\right)} \leq & N-m+1+\frac{m \varepsilon-\left(1-(1-\varepsilon)^{m}\right)}{\varepsilon\left(1-(1-\varepsilon)^{m}\right)} \\
1+\frac{\varepsilon N-\left(1-(1-\varepsilon)^{N}\right)}{\varepsilon\left(1-(1-\varepsilon)^{N}\right)} \leq & N-\sum_{i=1}^{\ell} m_{i}+1+\frac{\ell-1}{\varepsilon} \\
& +\sum_{i=1}^{\ell}\left[\frac{m_{i} \varepsilon-\left(1-(1-\varepsilon)^{m_{i}}\right)}{\varepsilon\left(1-(1-\varepsilon)^{m_{i}}\right)}\right]
\end{aligned}
$$

Note that, for $m_{i}$ such that $\sum_{i=1}^{\ell} m_{i}=N$, inequality (3.6) reduces to inequality (3.4). Therefore, we need to only focus on the last two inequalities, (3.5) and (3.6). Let us begin with the former. In order to prove (3.5), it is sufficient to show that

$$
\frac{N}{1-(1-\varepsilon)^{N}} \leq N-m+\frac{m}{1-(1-\varepsilon)^{m}} .
$$


Let $t=1-\varepsilon$, so that $t \in(0,1)$. Writing both sides over a common denominator, inequality (3.7) is equivalent to

$$
\frac{N t^{N}}{1-t^{N}} \leq \frac{m t^{m}}{1-t^{m}}
$$

Define the function $g(x):=x t^{x} /\left(1-t^{x}\right)$ for $x \geq 0$. Differentiating $g(x)$ gives

$$
\frac{\mathrm{d}}{\mathrm{d} x} g(x)=\frac{t^{x}\left(1-t^{x}+x \ln t\right)}{\left(1-t^{x}\right)^{2}} \text {. }
$$

It is straightforward to show that the denominator $\left(1-t^{x}\right)^{2}>0$ for all $x>0$. Also, $t^{x}>0$ for all $x \geq 0$. Define the function $h(x):=1-t^{x}+x \ln t$ for $x \geq 0$, and observe that $h(0)=0$ and

$$
\frac{\mathrm{d}}{\mathrm{d} x} h(x)=-t^{x} \ln t+\ln t=\ln t\left(1-t^{x}\right)<0,
$$

as $\ln t<0$ and $1-t^{x}>0$ for all $x>0$. Therefore, $h(x)$ is a decreasing function and $h(x)<0$ for $x>0$. This implies that $\mathrm{d} g(x) / \mathrm{d} x<0$ for $x>0$ and, consequently, $g(x)$ is a decreasing function. As $N>m$, we obtain (3.8) and subsequently (3.5).

Now let us consider inequality (3.6). In order to prove (3.6), it is sufficient to show that

$$
\frac{N}{1-(1-\varepsilon)^{N}} \leq N-\sum_{i=1}^{\ell} m_{i}+\frac{\ell-1}{\varepsilon}+\sum_{i=1}^{\ell}\left[\frac{m_{i}}{1-(1-\varepsilon)^{m_{i}}}-\frac{1}{\varepsilon}\right],
$$

which is equivalent to

$$
\frac{N(1-\varepsilon)^{N}}{1-(1-\varepsilon)^{N}}+\frac{1}{\varepsilon} \leq \frac{m_{1}(1-\varepsilon)^{m_{1}}}{1-(1-\varepsilon)^{m_{1}}}+\sum_{i=2}^{\ell}\left[\frac{m_{i}(1-\varepsilon)^{m_{i}}}{1-(1-\varepsilon)^{m_{i}}}\right] .
$$

As we have proved that $g(x)$ is a decreasing function for $x>0$,

$$
\frac{N(1-\varepsilon)^{N}}{1-(1-\varepsilon)^{N}} \leq \frac{m_{1}(1-\varepsilon)^{m_{1}}}{1-(1-\varepsilon)^{m_{1}}} \text {. }
$$

In order to obtain (3.9), we only need

$$
\frac{1}{\varepsilon} \leq \frac{m_{i}(1-\varepsilon)^{m_{i}}}{1-(1-\varepsilon)^{m_{i}}}
$$

or, equivalently,

$$
0 \leq \varepsilon m_{i}(1-\varepsilon)^{m_{i}}-\left(1-(1-\varepsilon)^{m_{i}}\right)=\left(1+\varepsilon m_{i}\right)(1-\varepsilon)^{m_{i}}-1
$$

for any $i=2, \ldots, \ell$. Define $f_{i}(x):=\left(1+x m_{i}\right)(1-x)^{m_{i}}-1$ for $i=1, \ldots, \ell$ and $x \in[0,1)$. We observe that $f_{i}(0)=0$, and differentiating $f_{i}(x)$ gives

$$
\begin{aligned}
\frac{\mathrm{d}}{\mathrm{d} x} f(x) & =m_{i}(1-x)^{m_{i}}-m_{i}\left(1+x m_{i}\right)(1-x)^{m_{i}-1} \\
& =m_{i}(1-x)^{m_{i}-1}\left[(1-x)-\left(1+x m_{i}\right)\right] \\
& =x m_{i}(1-x)^{m_{i}-1}\left(m_{i}-1\right) \\
& >0,
\end{aligned}
$$

as $2 \leq m_{i} \leq N-2$ for all $x \in(0,1)$. This implies that $f_{i}(x)$ is an increasing function, and $f_{i}(x)>0$ for $x \in(0,1)$. This gives (3.10) and subsequently (3.6). 


\section{Acknowledgements}

The authors gratefully acknowledge the support of the Netherlands Organisation for Scientific Research (NWO), under Meervoud grant number 632.002.401, the Australian Research Council Discovery Grant DP0666632, the Australian Research Council Linkage International Grants LX0560049 and LX0881972, and the Australian Research Council Centre of Excellence in the Mathematics and Statistics of Complex Systems. The authors are indebted to an anonymous referee and J. A. Filar for many insightful comments and discussions.

\section{References}

[1] Andramonov, M., Filar, J., Pardalos, P. and Rubinov, A. (2000). Hamiltonian cycle problem via Markov chains and min-type approaches. In Approximation and Complexity in Numerical Optimization, ed. P. Pardalos, Kluwer, Dordrecht, pp. 31-47.

[2] Borkar, V. S., Ejov, V. and Filar, J. A. (2004). Directed graphs, Hamiltonicity and doubly stochastic matrices. Random Structures Algorithms 25, 376-395.

[3] Borkar, V. S., Ejov, V. and Filar, J. A. (2009). On the Hamiltonicity gap and doubly stochastic matrices. Random Structures Algorithms 34, 502-519.

[4] Brin, S. And Page, L. (1998). The anatomy of a large-scale hypertextual Web search engine. Comput. Networks ISDN Systems 30, 107-117.

[5] EJov, V. ANd NGUYEN, G. T. (2009). Consistent behavior of certain perturbed determinants induced by graphs. Linear Algebra Appl. 431, 543-552.

[6] EJov, V., Filar, J. and Gondzio, J. (2004). An interior point heuristic for the Hamiltonian cycle problem via Markov decision processes. J. Global Optimization 29, 315-334.

[7] Ejov, V., Filar, J. A. AND NGuYen, M.-T. (2004). Hamiltonian cycles and singularly perturbed Markov chains. Math. Operat. Res. 29, 114-131.

[8] Ejov, V., Filar, J. A., Haythorpe, M. and Nguyen, G. T. (2009). Refined MDP-based branch-and-fix algorithm for the Hamiltonian cycle problem. Math. Operat. Res. 34, 758-768.

[9] Ejov, V., Filar, J., Murray, W. and Nguyen, G. T. (2008). Determinants and longest cycles of graphs. SIAM J. Discrete Math. 22, 1215-1225.

[10] Ejov, V., Litvak, N., Nguyen, G. T. and Taylor, P. G. (2008). Proof of the Hamiltonicity-trace conjecture for singularly perturbed Markov chains. Preprint.

[11] Filar, J. A. ANd Krass, D. (1994). Hamiltonian cycles and Markov chains. Math. Operat. Res. 19, $223-237$.

[12] Filar, J. A. ANd Lasserre, J. B. (2000). A non-standard branch and bound method for the Hamiltonian cycle problem. ANZIAM J. 42, C586-C607.

[13] Grinstead, C. M. And Snell, J. L. (1997). Introduction to Probability, 2nd edn. American Mathematical Society, Providence, RI.

[14] Haythorpe, M. (2010). Interior point and other algorithms for solving the Hamiltonian cycle problem. Doctoral Thesis, University of South Australia.

[15] Langville, A. N. And Meyer, C. D. (2006). Google's PageRank and Beyond: The Science of Search Engine Rankings. Princeton University Press, Princeton, NY.

[16] Litvak, N. And EJov, V. (2009). Markov chains and optimality of the Hamiltonian cycle. Math. Operat. Res. 34, 71-82.

[17] Seneta, E. (1981). Nonnegative Matrices and Markov Chains, 2nd edn. Springer, New York.

[18] Serra-Capizzano, S. (2005). Jordan canonical form of the Google matrix: a potential contribution to the PageRank computation. SIAM J. Matrix Anal. Appl. 27, 305-312.

[19] Volkovich, Y. V. (2009). Stochastic analysis of web page ranking. Doctoral Thesis, University of Twente. 